\title{
Cerebral Metabolism in the Management of TBI Patients
}

\author{
Roukoz B. Chamoun, Shankar P. Gopinath and Claudia S. Robertson*
}

Department of Neurosurgery, Baylor College of Medicine, Houston, Texas, USA

\begin{abstract}
Management after traumatic brain injury (TBI) is essentially directed at preventing and treating secondary brain insults. Ischemia has been traditionally recognized as a common and potentially devastating event after TBI. This has led to the development of management strategies aiming at optimizing blood flow and cerebral perfusion pressure. However, this school of thought has been challenged by data suggesting that ischemic injury is rather rare in TBI; the decrease in cerebral blood flow (CBF) being explained by a decrease in the metabolic demands, and the metabolic changes observed (hyperglycolysis and increase in lactate) being attributed to mitochondrial dysfunction. To date, despite significant progress in diagnostic and monitoring techniques, the role of ischemia remains a matter of debate. Cerebral metabolism is therefore key to making a therapeutic decision about treatment of cerebral hypoperfusion. Raising CBF may be beneficial if ischemia is present, but will not help if CBF is low because cerebral metabolism is suppressed. Further studies are still needed to understand the underlying mechanisms of metabolic and blood flow changes in TBI; but progress in this field could potentially have a significant impact on the optimal management strategy that needs to be adopted in severe TBI.
\end{abstract}

Keywords: Traumatic brain injury, cerebral blood flow, ischemia.

\section{INTRODUCTION}

Traumatic brain Injury (TBI) continues to represent the leading cause of mortality and morbidity in young adults. Due to the significant and potentially devastating impact on the individuals, their families and even the whole society, a large number of experimental and clinical studies have been conducted in order to better understand its pathophysiology. Expanding our knowledge on the molecular events of TBI is undoubtedly a crucial step in refining our management strategy and achieving a better patients' outcome.

The mechanisms of TBI are usually divided into primary and secondary insults. While primary insults represent the damage occurring at the moment of the impact, secondary insults refer to the complex cellular processes and biochemical cascades that occur in a delayed fashion. One of the key factors of post-traumatic secondary insults is related to changes in cerebral blood flow $(\mathrm{CBF})$ and to ischemia. A number of techniques, including brain oxygenation and microdialysis, have become available for monitoring aspects of brain metabolism in the intensive care unit. The purpose of this paper is to review the current knowledge regarding CBF, cerebral metabolism, and ischemia in TBI, and their potential impact on patients' management.

\section{METABOLIC EVIDENCE FOR ISCHEMIA IN TBI}

In 1978, Graham et al. [1] performed a comprehensive neuropathological and neurohistological examination on 151 patients who died as a result of a non-missile head injury. They identified ischemic damage in $91 \%$ of the cases even after excluding infarctions related to contusions and those of the brainstem of the type usually associated with raised

*Address correspondence to this author at the Department of Neurosurgery, Baylor College of Medicine, Houston, Texas, USA; Tel: 713-798-4696; Fax: 713-798-3739; E-mail: claudiar@bcm.tmc.edu intracranial pressure. Using Xenon CT, Bouma et al. [2] showed that CBF was low in the first 6 hours after severe TBI and then increased significantly. In one third of the patients it was less than $18 \mathrm{ml} / 100 \mathrm{mg} / \mathrm{min}$ which was considered as the threshold for ischemia. A significant correlation was found between $\mathrm{CBF}$ and outcome, with values of $18 \mathrm{ml} / 100 \mathrm{mg} / \mathrm{min}$ or less being associated with higher mortality and morbidity. The authors also found that the arteriovenous oxygen difference $\left(\mathrm{AVDO}_{2}\right)$ was initially high and then decreased over time. They concluded that the early changes were suggestive of ischemia (low CBF and high $\mathrm{AVDO}_{2}$ ) while delayed ones were related to low oxidative metabolism (low $\mathrm{AVDO}_{2}$ ) and relative hyperemia. Using the lactate-oxygen index (LOI), Sahuquillo et al. [3] found that $46 \%$ of their patients had a pattern consistent with ischemia during the first 24 hours after the accident. The LOI which equal the arteriovenous difference of lactate content (AVDL) divided by $\mathrm{AVDO}_{2}$ was in fact found to be a reliable estimate of $\mathrm{CBF}$, and patients with ischemia found to have a LOI of 0.08 or greater with a variable $\mathrm{AVDO}_{2}$ [4]. These findings pointed to a high prevalence of ischemia in TBI. The ischemic injury appeared as such to be the most important secondary insult with a major impact on prognosis. The assumption that ischemia is a major contributor to brain damage after TBI has led to a traditional management focusing on improving cerebral perfusion and blood flow.

\section{METABOLIC EVIDENCE AGAINST ISCHEMIA IN TBI}

Obrist et al. [5] used Xenon CT to measure CBF in 75 patients who were comatose as a result of their TBI. The measurements were done within 96 hours of the injury. They found low $\mathrm{CBF}$ in $45 \%$ of the cases and hyperemia in $55 \%$. However, patients with low $\mathrm{CBF}$ had no evidence of ischemia (due to reduced cerebral metabolic rate of oxygen $\mathrm{CMRO}_{2}$ and absence of wide $\mathrm{AVDO}_{2}$ ), but instead they showed expected coupling of $\mathrm{CBF}$ and metabolism. On the 
other hand, patients with hyperemia had narrow $\mathrm{AVDO}_{2}$ speaking for luxury perfusion (excessive blood flow relative to the metabolic requirements); this was highly associated with elevated intracranial pressure (ICP). Understanding the changes in cerebral metabolism following TBI is in fact essential for accurate interpretation of changes in CBF. A number of studies have recently allowed elucidating at least some aspects of these metabolic changes. Glenn et al. [6] found that compared to normal subjects, patients with TBI had significant depression in $\mathrm{CMRO}_{2}, \mathrm{AVDO}_{2}$, and to a lesser extent CMRglc (cerebral metabolic rate of glucose). Despite a significant elevation in mean lactate level, evidence of ischemia was not seen. In $76 \%$ of the patients at least one episode of brain uptake of lactate (measured by AVDlac: arteriovenous difference in lactate) was documented. Relative hyperglycolysis was also very common in this study. In accordance with data from other studies [7-9], these findings suggest that under certain conditions the brain can use lactate as a source of fuel. Interestingly, animal studies showed that TBI results in significant mitochondrial dysfunction with subsequent inhibition of the respiratory chainlinked electron transfer and energy transduction [10]. One would logically expect that such a dysfunction would have marked and complex effects on mitochondrial and cellular energy metabolism. In accordance with these findings, a more recent [11] clinical study found severe metabolic depression with reduction of $\mathrm{CMRO}_{2}$ and a less profound decrease in CMRglc. CBF analysis showed only moderate reduction in cerebral perfusion and the authors argued that cerebral metabolic failure after TBI is a common finding that is not of ischemic origin in most instances.

\section{POSITRON EMISSION TOMOGRAPHY (PET) STUD- IES}

Progress in our understanding of the metabolic changes that occur after TBI in humans has been possible through the recent use of PET scan in studying these patients. Bergsneider et al. [12] were able to document the occurrence of posttraumatic hyperglycolysis in human subjects. This was observed both globally and regionally. Hyperglycolysis was defined as an abnormal cellular state of increased glucose metabolism relative to the rate of oxygen utilization. It represents a nonphysiological uncoupling between glycolytic and oxidative metabolism.

However the role of ischemia after TBI remained a matter of debate due to conflicting results reported by different groups. Using PET techniques, an increased oxygen extraction fraction $(\mathrm{OEF})$ is used to indicate that $\mathrm{CBF}$ is reduced relative to cerebral metabolic needs. While Abate et al. [13] reported data consistent with "misery perfusion" with reduction in CBF and increase in OEF, Vespa et al. [14] found only $1 \%$ incidence of ischemia across all voxels as measured by $\mathrm{OEF}$, they concluded that the elevated lactate/pyruvate ratio that they observed in microdialysis represent a state of metabolic crisis but not related to ischemia; the authors suggested that such a metabolic crisis is likely related to mitochondrial dysfunction. Using combined microdialysis and PET techniques to examine cerebral metabolism, Hutchinson et al. [15] found that an increased microdialysate lactate/pyruvate ratio correlated with an increase in OEF. Menon et al. [16] have reported argued that OEF may not accurately reflect the presence of tissue hypoxia after TBI be- cause increased diffusion barriers may reduce the ability of the brain to increase oxygen extraction in response to hypoperfusion.

By examining changes in energy substrates and metabolites using microdialysis during periods of global ischemia induced by hypotension or increased intracranial pressure, Hlatky et al. [17] were able to identify patterns of early ischemia (depletion of energy substrates such as oxygen, glucose, pyruvate) and later ischemia (markedly increased lactate, increased lactate/pyruvate ratio, and increased glutamate). Decrease in tissue $\mathrm{pO}_{2}$ was the earliest sign of cerebral hypoperfusion, followed closely by decreases in microdialysate glucose and pyruvate. These patterns may be helpful in following patients over time, and in making decisions about whether therapies to raise CBF might be clinically useful in individual patients.

\section{TECHNICAL LIMITATIONS}

Although brain oxygenaion, microdialysis, Xenon CT and PET scan have proved to be valuable tools in exploring the underlying metabolic changes in TBI, these techniques suffer from a number of limitations possibly explaining some of the divergence in the results obtained by different groups. While some of the methods used (such as jugular venous oxygen saturation) detect globally averaged values, others (such as microdialysis and brain tissue $\mathrm{pO}_{2}$ probes) are limited to highly localized areas. By allowing measurements of $\mathrm{CBF}, \mathrm{CMRO}_{2}$ and OEF, PET scan quickly emerged as the preferred techniques. However, radiation exposure and the need to transfer of the patients to perform the study (as opposed to bed side monitoring techniques) have limited the possibility of obtaining serial values [18]. As a result, the data analyzed is limited to a small period of time during which data acquisition is obtained, while the relationship between CBF and metabolism might change significantly over time in TBI. The analysis is also dependent on regions of interests (ROI) used while TBI can be very heterogeneous in nature.

\section{CONCLUSIONS}

Understanding the cause of cerebral hypoperfusion and making a decision to treat a reduced CBF is complex. Evidence of a reduced brain oxygenation, either increased jugular venous oxygen saturation or a reduced brain tissue $\mathrm{pO}_{2}$ especially if coupled with an increased microdialysate lactate/pyruvate ratio, favor the presence of ischemia and suggest that a treatment to improve cerebral oxygenation or blood flow could be beneficial. These changes in cerebral metabolism also provide a way to follow patients to assess the effects of treatment. As a conclusion the role of ischemia in TBI continues to fuel a lot of debate. Because of its potential major implication on management strategies of patients with severe TBI, further studies are clearly still needed in this area.

\section{ACKNOWLEDGEMENT}

This work was supported by NIH grant \#P01-NS38660.

\section{REFERENCES}

[1] Graham DI, Adams JH, Doyle D. Ischaemic brain damage in fatal non-missile brain injuries. J Neurol Sci 1978; 39: 213-34. 
[2] Bouma GJ, Muizelaar JP, Choi SC, Newlon PG, Young HF. Cerebral blood flow and metabolism after severe traumatic brain injury: the elusive role of ischemia. J Neurosurg 1991; 75: 685-93.

[3] Sahuquillo J, Poca MA, Garnacho A, et al. Early ischaemia after severe head injury. Preliminary results in patients with diffuse brain injuries. Acta Neurochir (Wien) 1993; 122(3-4): 204-14.

[4] Robertson CS, Narayan RK, Gokaslan Z, et al. Cerebral arteriovenous oxygen difference as an estimate of cerebral blood flow in comatose patients. J Neurosurg 1989; 70: 222-30.

[5] Obrist WD, Langfitt T, Jaggi J, Cruz J, Gennarelli TA. Cerebral blood flow and metabolism in comatose patients with acute head injury. Relationship to intracranial hypertension. J Neurosurg 1984; 61: 241-53.

[6] Glenn TC, Kelly DF, Boscardin WJ, et al. Energy dysfunction as a predictor of outcome after moderate or severe head injury: indices of oxygen, glucose, and lactate metabolism. J Cereb Blood Flow Metab 2003; 23(10): 1239-50.

[7] Ros J, Pecinska N, Alessandri B, Landolt H, Fillenz M. Lactate reduces glutamate-induced neurotoxicity in rat cortex. J Neurosci Res 2001; 66(5): 790-94.

[8] Chen T, Qian YZ, Di X, Rice A, Zhu JP, Bullock R. Lactate/glucose dynamics after rat fluid percussion brain injury. J Neurotrauma $2000 ; 17(2)$ : $135-42$.

[9] Magistretti PJ, Pellerin L, Rothman DL, Shulman RG. Energy on demand. Science 1999; 283(5401): 496-97.

[10] Xiong Y, Gu Q, Peterson PL, Muizelaar JP, Lee CP. Mitochondrial dysfunction and calcium perturbation induced by traumatic brain injury. J Neurotrauma 1997; 14(1): 23-34.
[11] Soustiel JF, Sviri GE. Monitoring of cerebral metabolism: nonischemic impairment of oxidative metabolism following severe traumatic brain injury. Neurol Res 2007; 29(7): 654-60.

[12] Bergsneider M, Hovda DA, Shalmon E, et al. Cerebral hyperglycolysis following severe traumatic brain injury in humans: a positron emission tomography study. J Neurosurg 1997; 86: 241-51.

[13] Abate MG, Trivedi M, Fryer TD et al. Early derangements in oxygen and glucose metabolism following head injury: the ischemic penumbra and pathophysiological heterogeneity. Neurocrit Care 2008; 9(3): 319-25.

[14] Vespa P, Bergsneider M, Hattori N et al. Metabolic crisis without brain ischemia is common after traumatic brain injury: a combined microdialysis and positron emission tomography study. J Cereb Blood Flow Metab 2005; 25(6): 763-74.

[15] Hutchinson PJ, Gupta AK, Fryer TF et al. Correlation between cerebral blood flow, substrate delivery, and metabolism in head injury: a combined microdialysis and triple oxygen positron emission tomography study. J Cereb Blood Flow Metab 2002; 22(6): 735-45.

[16] Menon DK, Coles JP, Gupta AK, et al. Diffusion limited oxygen delivery following head injury. Crit Care Med 2004; 32(6): 138490 .

[17] Hlatky R, Valadka AB, Goodman JC, Contant CF, Robertson CS. Patterns of energy substrates during ischemia measured in the brain by microdialysis. J Neurotrauma 2004; 21(7): 894-906.

[18] Menon DK. Brain ischaemia after traumatic brain injury: lessons from $15 \mathrm{O} 2$ positron emission tomography. Curr Opin Crit Care 2006; 12(2): 85-9.

(c) Chamoun et al.; Licensee Bentham Open.

This is an open access article licensed under the terms of the Creative Commons Attribution Non-Commercial License (http://creativecommons.org/licenses/by-nc/3.0/) which permits unrestricted, non-commercial use, distribution and reproduction in any medium, provided the work is properly cited. 